\title{
On the Cauchy Problem of the Relativistic Boltzmann Equation*
}

\author{
KLAUS BichteleR** \\ Southwest Center for Advanced Studies \\ Received January 29, 1967
}

Abstract. It is shown that the relativistic Boltzmann equation has a local solution through an initial distribution function, if the scattering cross section is bounded for high energies and if the initial distribution falls off exponentially with the energy.

\section{Resume of Relativistic Gas Kinetics and Notation ${ }^{1}$}

Within the framework of general relativity, a gravitational field is described by a 4-dimensional manifold $M$ together with a metric $G_{a b}(x)$ of signature $(+1,-1,-1,-1)$. The phase space of a particle is the tangent bundle $T(M)$ of $M$. If $x^{a},(a=0,1,2,3)$, is a coordinate system in $M$, and if $T$ is a tangent to $M$ at a point $x$ in the domain of $x^{a}$, then $T$ can be written

$$
T=\left.p^{a} \frac{\partial}{\partial x^{a}}\right|_{x}
$$

and $T \rightarrow\left(x^{a}, p^{a}\right)$ is a coordinate system in $T(M)$, called the coordinate system associated with $x^{a}$. The set of all coordinate systems in $T(M)$ so obtained defines on $T(M)$ a differentiable structure which turns $T(M)$ into a differentiable manifold of dimension 8 . A particle is represented as a path $\left(x^{a}(t), p^{a}(t)\right)$ in $T(M)$, where $x^{a}(t)$ describes the position of the particle for the parameter-time $t$, and $p^{a}(t)$ is its four-momentum at that instant. If we choose the parameter $t$ such that $\frac{\partial x^{a}}{\partial t}=p^{a}$ then the tangent of the phase path of a test particle moving under the influence of an external electromagnetic field $F_{b}^{a}(x)$ and gravitational field $G_{a b}(x)$ is

$$
X=p^{a} \frac{\partial}{\partial x^{a}}+\left\{e F_{b}^{a}(x) p^{b}-\Gamma_{b c}^{a} p^{b} p^{b}\right\} \frac{\partial}{\partial p^{a}} .
$$

* Supported by the National Aeronautics and Space Administration under Grant No. NGR 44-004-042.

** On leave from the University of Heidelberg.

1 For this paragraph cf. Bichteler (1965). For different approaches, see Chernikov (1962), TAUber-Weinberg (1961), Lindquist (1966). 
Thus, $X$ is a tangent field to the phase space $T(M)$, the integral curves of which are the possible phase paths of particles with charge $e$, falling freely in a combined gravitational and electromagnetic field. A point $\left(x^{\prime}, p^{\prime}\right)$ on the unique integral curve of $X$ through $(x, p)$ is called earlier than $(x, p)$, if it has smaller parameter value: $t^{\prime}<t$.

With the metric given, the phase space carries the following additional structures, which will be needed in the sequel: As

$$
d X=\sqrt{-\operatorname{det}\left(G_{a b}\right)} d x^{0} \wedge d x^{1} \wedge d x^{2} \wedge d x^{3}
$$

is a coordinate-independent 4-form on $M$ - the 4-volume element - so

$$
d_{x} P=\sqrt{-\operatorname{det}\left(G_{a b}(x)\right)} d p^{0} \wedge d p^{1} \wedge d p^{2} \wedge d p^{3}
$$

is a coordinate-independent measure on the fiber $T_{x}$ of $T(M)$ in $x$, often abbreviated $d P$. Also,

$$
d \tau=d X \wedge d_{x} P
$$

is a coordinate-independent 8-form (measure) on the phase space $T(M)$.

As in Newtonian gas kinetics, the density of phase paths actually occupied by the particles of a gas is described by a function $F$ on the phase space in the following manner: If $H$ is a piece of a seven dimensional hypersurface in $T(M)$, then

$$
\int_{H} F \omega=\nu(H)
$$

is the number of particles crossing $H$. Here

$$
\begin{gathered}
\omega=p^{a} \eta_{a b c d} d x^{b} \wedge d x^{c} \wedge d x^{d} \wedge d_{x} P+ \\
+\left\{F^{a}{ }_{b} p^{b}-\Gamma_{b c}^{a} p^{b} p^{c}\right\} \eta_{a b c d} d p^{b} \wedge d p^{c} \wedge d p^{d} \wedge d X
\end{gathered}
$$

is a 7-form measuring the size $\int_{H} \omega$ of $H^{2}$. $\omega$ is carefully selected from all possible measures for the size of $H$ by requiring it to satisfy

$$
d \omega=0, d \tau=d t \wedge d \omega, \int_{H} \omega=0 \quad \text { whenever } X \text { is tangent to } H .
$$

The properties (5) are analogous to those of Lebesgue-measure in Newtonian phase space; in particular, they imply Liouville's theorem.

If all the particles of the gas are of the same mass $m$ then all possible phase paths lie in the subbundle $\left\{(x p): p^{a} p^{b} G_{a b}(x)=m^{2}\right\}$ of $T(M)$, which is a 7-dimensional submanifold of $T(M)$ called the mass hyperboloid $\Pi^{m}(M)$. The tangent field $X$ is tangent to $\Pi^{m}(M)$, hence is a tangent field on $\Pi^{m}(M)$, and the parameter $t$ equals eigentime $/ m$ (and is an affine parameter if $m=0$; for in that case empirically $e=0$ ). If we replace $T(M)$ by $\Pi^{m}(M)$ in the preceding paragraphs then all considera-

\footnotetext{
${ }^{2} \eta_{a b c d}$ is the totally skew tensor density with $\eta_{0123}=\sqrt{-\operatorname{det}\left(G_{a b}\right)}$.
} 
tions still go through, with the dimensions lowered by one. In particular, the fiber $\Pi_{x}^{m}$ has a coordinate-independent measure

$$
d_{x}^{3} P=d_{x} P \delta\left(p^{2}-m^{2}\right),
$$

again abbreviated $d P$. By $d \tau$ we understand now $d X \wedge d_{x}^{3} P$, which is a measure on $\Pi^{m}(M) \cdot v(H)$ describes the flow of paths in $\Pi^{m}(M)$ through a six-dimensional hypersurface $H$ and is of the form $F \omega$, where $\omega$ satisfies (5) on $\Pi^{m}(M)$.

With the notations as in the last paragraph, the relativistic Boltzmann equation reads

$$
\begin{aligned}
(X F)(x p)= & \iiint\left\{F\left(x p_{3}\right) F\left(x p_{4}\right)-F(x p) F\left(x p_{2}\right)\right\} \times \\
& \times W\left(p p_{2} \rightarrow p_{3} p_{4}\right) \delta\left(p+p_{2}-p_{3}-p_{4}\right) d P_{2} d P_{3} d P_{4}
\end{aligned}
$$

with

$$
W\left(p_{1} p_{2} \rightarrow p_{3} p_{4}\right)=k\left(p_{1} p_{2}\right) \sigma\left(p_{1} p_{2} \rightarrow p_{3} p_{4}\right)
$$

where $k\left(p_{1}, p_{2}\right)=\sqrt{\left(p_{1 a} p_{2}\right)^{2}-m^{4}}=1 / 2\left|p_{1}-p_{2}\right|\left|p_{1}+p_{2}\right|$ is a kinematical factor and

$$
\sigma(12 \rightarrow 34)=\sigma(21 \rightarrow 34)=\sigma(34 \rightarrow 12)
$$

is the Lorentz-invariant cross section ${ }^{3}$ for collisions $p_{1} p_{2} \rightarrow p_{3} p_{4} . \sigma$ is a function defined on the collision manifold ${ }^{4}$, that is, on the set of all quadruples $(1,2,3,4)$ of tangents in $x$ with $1^{a}+2^{a}=3^{a}+4^{a}$. The $\delta$-factor in (6) takes care of the 4-momentum conservation and has to be understood mathematically in the sense of GELFAND and SHILOv (1960); the orientation of the collision manifold is chosen such that $\delta d P_{2} d P_{3} d P_{4}$ is a positive measure.

Equation (6) was apparently first given by LICHNERowicz and MARRot, 1940. As it stands, it describes a gas in which only elastic collisions take place. This equation can easily be generalized such as to include quantum statistics, inelastic scattering such as decay and absorption, and gases which consist of $N$ components with fixed masses and charges ${ }^{5}$. However, the local existence theorem with which we shall be concerned holds for all of these generalizations, and as its proof is in principle the same, we shall confine ourselves to the simple equation (6). For further simplicity we assume $F_{b}^{a}=0$ in the sequel. The existence theorem for the most general Boltzmann equation requires only minor and obvious alterations of the proof below.

We call a solution of (6) any function $F$, defined in the tangent bundle of a measurable subset of $M$ for which both sides of (6) exist in the usual sense almost everywhere (a.e.) with respect to the measure $d \tau$ and coincide a.e. For the left hand side $X F$ of (6) this means that the

\footnotetext{
${ }^{3}$ Some authors call the differential $\sigma \delta d P_{3} d P_{4}$ the cross section.

4 This notion was apparently first introduced by EHLERS (1961). 1, 2, $1^{a}, 3^{a} \ldots$ stands for $p_{1}, p_{2}, p_{1}{ }^{a}, p_{2}{ }^{a} \ldots$ in the sequel.

5 Cf. e.g. Bichteler (1965).
} 
derivative of $F$ along the integral curves of $X$ exists at almost all points of the domain of $F$ (the partial derivatives of $F$ which occur in the representation (1) of $X$ need not exist).

\section{Statement of the Theorem}

Suppose we are given a spacelike 3-dimensional hypersurface of $M$ and a compact connected subset $H$ of it. We assume that $H$ is equal to the closure of its interior $\dot{H}$ in the topology of the hypersurface. One may visualize $H$ as a homeomorphic image in $M$ of the 3 -dimensional unit ball. We define the restricted domain of dependence of $H$ as the set $D$ of all points $x$ of $M$ such that any particle through $x$ which moves according to (1) was earlier in $H$ (cf. Fig. 1). In other words, $D$ is the set of all points $x \in M$ such that for any $p \in T_{x}$ the unique integral curve of $X$ through $(x, p)$ contains an earlier point $\left(x^{\prime}, p^{\prime}\right)$ with $x^{\prime} \in H$. For any $(x, p), x \in D$, we denote by $\left(x^{\prime}, p^{\prime}\right)$ the latest such point.

$D$ is a closed subset of $M$. For let $x_{n} \in D$ converge to $x$ and let $p \in T_{x}$. We may without loss of generality assume that the $x_{n}$ and $x$ lie in a coordinate domain and consider the integral curves of $X$ through $\left(x_{n}, p_{n}\right)$ where $p_{n}$ has the same coordinates as $p$ in the associated coordinate system (cf. Sec. I). According to the well-known theorem on the dependence of integral curves on the initial point ${ }^{6}$, the integral curves through $\left(x_{n}, p_{n}\right)$ converge uniformly to the one through $(x, p)$ for bounded values of the parameter $t$. In particular, $\left(x_{n}^{\prime}, p_{n}^{\prime}\right)$ converges to a point $\left(x^{\prime}, p^{\prime}\right)$ on the integral curve through $(x, p)$. Thus $x^{\prime} \in H$ since $H$ is closed. So, recalling the definition of $D$, we see that $x \in D$ and therefore $D$ is closed and hence measurable?

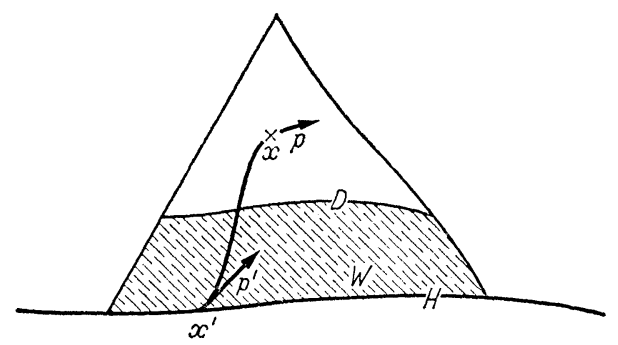

Fig. 1. The restricted domain of dependence $D$ and a time layer $W$

${ }^{6}$ Cf. e.g., KaMKE (1956). We assume $G_{a b}$ and $F^{a}{ }_{b}$ of class $C^{2}$.

7 One shows by a similar argument that the subset $\stackrel{D}{D}$ of $D$ consisting of all $x \in D$ such that $x^{\prime} \in \stackrel{\circ}{H}$ for all $p \in T_{x}$ is an open subset of $M$ and closure $(\stackrel{\circ}{D})=D$. We shall not need this fact. It shows, however, that $D$ is a "sensible" set, adequately visualized as in Fig. 1. 
Let us finally define a "time layer over $H$ ". By this we mean an open subset (in the relative topology) $W$ of $D$ which contains $H$ (cf. Fig. 1). The reason for this definition of $D$ and $W$ is the following: We assume we are given an initial distribution $f$ on the part of $T(M)$ which lies over $H$ and which we shall denote by $H \times T$. Then we cannot hope to make a uniqueness statement for a solution $F$ of (6) through $f$ on points outside $D$. For such an $x$ can be reached by particles starting outside $H$. And so the distribution may be influenced differently by different initial distributions outside $H$.

Theorem. Let $f$ be a function on $H \times T$, measurable with respect to $\omega$ and "exponentially bounded". That means we assume there exists a constant $A$ and a continuous vector field $\beta_{a}^{\prime}(x)>0^{8}$ on $H$ such that

$$
|f(x p)| \leqq A e^{-\beta_{a}^{\prime}(x) p^{a}}, x \in H, \quad p \in T_{x} .
$$

Furthermore, let the cross section be a continuous function on the collision manifold such that the total cross section is bounded:

$$
\sigma(E):=\int \sigma(12 \rightarrow 34) \delta(1+2-3-4) d 3 d 4 \leqq \sigma=\text { const . }
$$

Then there exists a time layer $W$ over $H$ and a solution $F$ of (6) on $W$ which is again exponentially bounded. Any two such solutions are equal a.e. Moreover, if $f \geqq 0$, then also $F \geqq 0$.

Remarks. 1 . The uniqueness statement of the theorem is to be understood in terms of the class of all exponentially bounded functions: If $F, F^{\prime}$ are solutions of (6) in $W$ and are exponentially bounded, then $F=F^{\prime}$ a.e.

2. The requirement (9) on $f$ means that for the observer field $\beta^{a}(x)$ the number of particles with energy $E$ decreases exponentially with $E$. Then, incidentally, relative to any other observer field $\beta^{\prime}{ }^{a}(x)$ there also is exponential decrease, and, according to the theorem, will always remain so. The requirement (9) is not as restrictive as it may seem at first: All local equilibrium distribution functions are exponentially bounded $^{9}$ and so are distribution functions which are close to local equilibrium in the sense that their momenta are close to those of an equilibrium distribution. Furthermore, in the (still apparently mathematically not justified) Chapman-Enskog approximation, $F$ is written

$$
F=e^{-\beta_{a}(x) p^{a}}(1+\phi)
$$

with a small $\phi$, for which a linear integro-differential equation is derived. The theorem as stated above tells that $F$ remains in the form (11) if it was in that form initially, with $\phi$ bounded. This is of course necessary for the Chapman-Enskog method to make sense.

${ }^{8} \beta>0$ means that $\beta^{a}$ is pointing into the future; we say $\beta$ is positive.

9 Cf. Bichteler, 1964. 
3. Morgenstern, 1955, proved ${ }^{10}$ a global existence theorem in the non-relativistic case by a method which overcomes the difficulty of how to define both sides of (6) for a sufficiently large class of functions by modifying the collision term in (6) such that it can be applied to integrable functions $F$. Unfortunately, he must assume the total cross section $\sigma(E)$ to be $O(1 / E)$, and the field equations do not follow from his modified Boltzmann equation. His theorem can be generalized immediately to the general relativistic case.

\section{Proof of the Theorem}

The proof of the theorem is an application of the well-known Banach fixed point theorem which states that a mapping of a complete metric space into itself which uniformly decreases distances has a unique fixed point. Our proof proceeds as follows. As a first step we establish a lemma stating the existence of a continuous vector field $\beta_{a}(x)$ on $D$ which continues the given vector field $\beta_{a}^{\prime}$ on $H$ in a suitable way such that $-\beta_{a}(x) p^{a}$ can be used as an exponential bound for the prospective solution $F$. In the second step, we choose a time layer $W$ and consider the set $\Phi$ of all measurable functions $F$ on $W$, which are bounded by $2 A e^{-\beta_{a} p^{a}} . \Phi$ can be made into a complete metric space in a natural way. Then obviously a solution of (6) through $f$ in $\Phi$ is a fixed point of the mapping $\sim$ of $\Phi$ in $\Phi$ defined by

$$
\begin{gathered}
\tilde{F}(x p)=f\left(x^{\prime} p^{\prime}\right)+\int_{\left(x^{\prime} p^{\prime}\right)}^{(x p)} d t \iiint\left\{F\left(x_{t} 3\right) F^{\prime}\left(x_{t} 4\right)-\right. \\
\left.-F^{\prime}\left(x_{t} p_{t}\right) F^{\prime}\left(x_{t} 2\right)\right\} \delta\left(p_{t}+2-3-4\right) W(12 \rightarrow 34) d 2 d 3 d 4 ;
\end{gathered}
$$

and, conversely, every fixed point of $\sim$ is a solution of (6) through $f$. The main labor in the proof then consists in showing that the mapping $\sim$ is well defined for functions of $\Phi$, that it maps $\Phi$ into itself, and that it is uniformly contractive. Application of Banach's theorem then yields the existence and uniqueness of a solution $F$ of (6) through $f$.

Step 1. Lemma. Let $\beta^{\prime}{ }_{a}(x)$ be a covariant timelike vector field, defined and continuous on $H$ or on a compact time layer $K$ over $H$. Assume $\Gamma^{a}{ }_{b c}(x)$ to be of class $C^{1}$. Then there exists a continuous timelike covariant vector field $\beta_{a}(x)$ on $D$ such that:

(A) $\beta^{\prime}{ }_{a}(x) \geqq \beta_{a}(x)$ for all $x \in K$,

(B) The function $\beta_{a}(x) p^{a}$ on $D \times T$ does not increase along phase paths.

Let us clarify the statement (A). We call the closed (tangential) forward light cone in the point $x \in M, V_{x}$, and its open interior which consists of timelike vectors only, $V_{x}$. We call a vector $u_{a} \in T_{x}$ greater

10 Up to a minor oversight concerning the $\delta$-factor, which can be repaired by proceeding as in the proof below. 
than the vector $v_{a} \in T_{x}$, and we write $u_{a} \geqq v_{a}$, if $u_{a}-v_{a} \in V_{x}$. We say $u$ is strictly greater than $v$ if $u-v \in \dot{V}$. Thus $u_{a} \geqq v_{a}$ if and only if $u_{a} p^{a} \geqq v_{a} p^{a}$ for all $p \in V_{x}$. A vector $\beta_{a}(x)$ is called smaller than a set $B_{x}$ of vectors of $T_{x}$, if it is smaller than any vector of $B_{x}$. The relation $\geqq$ gives rise to an order on $T_{x}$ compatible with the vector space structure of $T_{x}$.

Proof of the Lemma. a) Let $B_{x}$ be a compact subset of $\dot{\circ}_{x}$. Then there exists a unique vector $\beta_{a}\left(B_{x}\right) \in V_{x}$ of greatest length, which is smaller than $B_{x}$. For, first of all, the set of vectors in $\stackrel{\circ}{V}_{x}$ smaller than $B_{x}$ is non void (cf. Fig. 2). Otherwise, $B_{x}-r t_{a}$ would contain points outside $V_{x}$ for arbitrary $t_{a} \in \stackrel{\circ}{x}_{x}$ and arbitrary $r>0$. Taking $t_{a}$ fixed and $r=1 / n(n=1,2 \ldots)$ there would exist a sequence $b_{n} \in B_{x}$ with $b_{n}-1 / n t_{a} \notin \stackrel{\circ}{V}_{x}$. By compactness of $B_{x}$ we may assume $b_{n}$ convergent to $b \in B_{x}$, so $b \notin \stackrel{\circ}{V}_{x}$ in contradiction to $B_{x} \subset \stackrel{\circ}{V}_{x}$. So the function $v_{a} \rightarrow v_{a} v^{a}$, restricted to the (compact) set of all vectors of $V_{x}$ smaller than $B_{x}$, takes on a positive maximum at a vector $\beta_{a}\left(B_{x}\right)$. If there were a second vector $\beta_{a}^{\prime}$ of greatest length smaller than $B_{x}$, then $1 / 2\left(\beta+\beta^{\prime}\right)$ would be a vector smaller than $B_{x}$ of length greater than that of $\beta$ and $\beta^{\prime}$. So, $\beta\left(B_{x}\right)$ is unique.

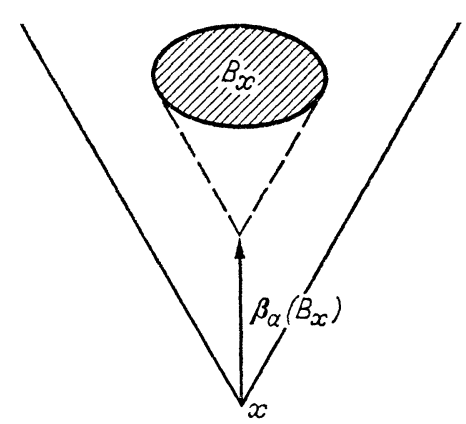

Fig. 2. The greatest vector smaller than $B_{x}$

b) The mapping $B_{x} \rightarrow \beta_{a}\left(B_{x}\right)$ has the following properties:

b1) $B_{x}^{\prime}<B_{x} \Rightarrow \beta_{a}\left(B_{x}^{\prime}\right) \geqq \beta_{a}\left(B_{x}\right)$,

which is immediate from its definition. Further, it is continuous in the following sense: For any $\varepsilon>0$ there exists a compact neighborhood $U$ of $B_{x}$, contained in $\dot{\circ}_{x}$ and such that

b2) $\beta_{a}\left(B_{x}^{\prime}\right)>\beta_{a}\left(B_{x}\right)(1-\varepsilon)$ for all $B_{x}^{\prime} \subset U$.

The argument is immediate. Let us remark that the family of sets of vectors which lie strictly between two given ones forms a basis of the topology of $\stackrel{\circ}{V}_{x}$. 
c) We assume temporarily for the next few steps that $D$ lies in a coordinate domain and that coordinates $x^{a}$ are chosen such that $x^{0}=0$ on $H$.

Let $x$ be a point of $D, x^{\prime}$ a point of $K$ earlier than $x$, and let $d$ be a broken piecewise time - or lightlike geodesic joining $x$ and $x^{\prime}$. Let $D_{x}$ be the set of all such broken geodesics. We define a vector $b_{x}(d)$ in $\stackrel{\circ}{V}_{x}$ by transferring the given vector $\beta^{\prime}{ }_{a}\left(x^{\prime}\right)$ in $\stackrel{\circ}{V}_{x^{\prime}}$ parallely from $x^{\prime}$ to $x$ along $d$, thus getting a map $b_{x}: D_{x} \rightarrow \stackrel{\circ}{V}_{x}$.

If we parametrize the curves of $D_{x}$ by the coordinate time $x^{0}$ and provide $D_{x}$ with the topology of uniform convergence in $x^{0}$, then $b_{x}$ is continuous. For, let us consider two broken geodesics $d$ and $d$ of $D_{x}$ with starting points $x^{\prime}$ and $\tilde{x}^{\prime}$. If we choose $d$ and $\tilde{d}$ sufficiently close to each other then the two following vectors of $V_{x}$ are arbitrarily close to each other: the vector $\beta^{\prime}{ }_{a}\left(x^{\prime}\right)$ after parallel transfer from $x^{\prime}$ along a geodesic to $\tilde{x}^{\prime}$ and from $\tilde{x}^{\prime}$ along $\tilde{d}$ to $x$; and the vector $\beta^{\prime}{ }_{a}\left(\tilde{x}^{\prime}\right)$ after parallel transfer to $x$ along $\tilde{d}$. This is an immediate consequence of the continuity of $\beta^{\prime}{ }_{a}$ and the smooth dependence of the solution of a differential equation (here the equ. of parallel transport) on its initial value. Thus, in order to prove continuity of $b_{x}$, it suffices to assume that the curves $d$ and $d$ have the same starting point $x^{\prime}$. We must show that if $d$ and $\tilde{d}$ are sufficiently close together, then it matters arbitrarily little, whether we transport $\beta^{\prime}{ }_{a}\left(x^{\prime}\right)$ along $d$ or along $d$ to $x$. Or, what amounts to the same, we must show that $\beta^{\prime}{ }_{a}\left(x^{\prime}\right)$, transported along $d$ to $x$ and then back to $x^{\prime}$ along $d$, is arbitrarily close to $\beta^{\prime}{ }_{a}\left(x^{\prime}\right)$. That this is so if the loop described by $d$ and $\tilde{d}$ is a small geodesic triangle $\Delta$, is a well-known fact (which can be used to define the Riemann-tensor; cf. Bishop and CRittenden (1960), Sec. 6.1.7). In that case $\beta_{a}^{\prime}\left(x^{\prime}\right)-\beta^{\prime}{ }_{a}\left(x^{\prime}\right)_{\text {transp. }}=O(|\Delta|)$ where $|\Delta|$ denotes the area of $\Delta$. But the latter estimate still holds for an arbitrary loop made of a broken geodesic, as can be seen by splitting it into a sequence of geodesic triangles (cf. Lichnerowicz, 1955, p. 51). Now if $d$ and $\tilde{d}$ are sufficiently close uniformly in $x^{0}$, then the area $\Delta$ enclosed can be made arbitrarily small; thus the continuity of $b_{x}$ is established.

We continue $b_{x}$ to a mapping, called again $b_{x}$, of the set $\bar{D}_{x}$ of all curves which are uniform limits of broken geodesics of $\bar{D}_{x}$, into $\dot{V}_{x}$. $\bar{D}_{x}$ consists of time- or lightlike continuous curves, and $b_{x}$ is continuous also on $\bar{D}_{x}$. Now $\bar{D}_{x}$ is compact in the topology of uniform convergence in $x^{0}$. For, $\bar{D}_{x}$ consists of continuous functions in a real interval, is equicontinuous since the (coordinate-) velocity of light is bounded in the coordinate domain, and every element of $\bar{D}_{x}$ takes its values in the intersection of $D$ with the backward light cone of $x$, which is compact. Thus, according to the theorem of Ascoli, $\bar{D}_{x}$ is compact in the topology of uniform convergence in $x^{0}$. 
Thus the set of $b_{x}\left(\bar{D}_{x}\right)=B_{x} \subset \dot{V}_{x}$ is compact as a continuous image of a compact set.

We are now in the situation a) and define the vector field $\beta_{a}(x)$ of the statement of the lemma by $\beta_{a}(x)=\beta_{a}\left(B_{x}\right)$.

d) Statement $(A)$ of the lemma is immediately obvious from the definition of $\beta_{a}$, and so is $(B)$. To prove the continuity of $\beta_{a}$, one repeats essentially the reasoning of $\mathrm{c}$ ), varying also $x^{11}$ and making use of the continuity property stated in b).

e) If $D$ is not contained in a coordinate domain, we define $\beta_{a}$ as above in coordinate patches containing $H$. Where they overlap, the $\beta$ 's are equal. We thus obtain $\beta$ on a time layer $L$ containing $H$. We continue $\beta$ by continuity to its closure $\bar{L}$ and proceed the same way again, now starting with the upper boundary of $\bar{L}, \bar{L}-L$, instead of $H$. Thus we obtain a vector field $\beta$ on $D$ which obviously meets the requirements of the lemma.

Note that $\beta_{a}$ coincides with $\beta^{\prime}{ }_{a}$ on $H$.

Step 2. a) Consider the integral $J(p, \beta)=\int_{2} p_{a} 2^{a} \cdot e^{-\beta 2} d 2$. Because of the exponential factor it is convergent and a smooth function of $p$ and $\beta$. Simple symmetry considerations show that it depends on these parameters in the form $J(p \beta)=\beta_{a} p^{a} Z(|\beta|)$ where $Z$ is a smooth function of the length $|\beta|$ of $\beta$. Further, if $x$ is a point of $D$ joined to a point $x^{\prime} \in H$ by a geodesic $\gamma$, we have $\int_{\gamma} I(p \beta) d t=\int_{x^{\prime}}^{x} Z(|\beta|) \beta_{a}(x) d x_{a}$, since $p^{a} d t=d x^{a}$ on $\gamma$ according to (1). Let us choose a number $B$, which we shall specify later according to our needs, and define a time layer $W$ by

$$
\int_{x^{\prime}}^{x} Z(|\beta|) \beta_{a}(x) d x^{a}<B
$$

The set of all $x$ such that (13) holds for all geodesics from $x$ to $H$ is, in fact, a time layer, since $x \rightarrow Z(|\beta|(x)) \beta_{a}(x) d x^{a}$ is a continuous form.

b) Let us denote by $\Phi$ the set of all real-valued functions $F$ on $W$ which are measurable with respect to $d \tau((4))$ and which satisfy

$$
|F(x p)| \leqq 2 A e^{-\beta_{a} p a} \quad \text { a.e. }
$$

We equip $\Phi$ with the following distance function \|\| :

$$
\|F-G\|=\text { ess. } \max . e^{+\beta_{a}(x) p^{a}}|F(x p)-G(x p)| .
$$

That is, $\|F-G\|$ is the infimum of all numbers $c$ such that the set of points $(x p)$ where $e^{\beta p}|F-G|$ exceeds $c$ has $d \tau$-measure zero. It is easy to check that \| $\|$ has the properties of a pseudometric and that $\Phi$ is complete. For, if $F_{n}$ is a Cauchy sequence under \|\| , then $e^{\beta p} F_{n}$ is a

11 For the details ef. Bichteler, 1965. 
Cauchy sequence in $L^{\infty}(W, d \tau)$, so converges to a function which is of the form $e^{\beta p} F, F \in \Phi$. And this means exactly that $F_{n} \rightarrow F$ in $\Phi$. Note that $\Phi$ is not separated, however.

c) Let us suppose for the moment (to be precise, until Section e where we shall prove it) that the integrals in (12) are well-defined. We may then estimate $\|\tilde{F}\|$ :

$$
\begin{aligned}
& \left|e^{\beta(x) p} F(x p)\right| \leqq\left|e^{\beta(x) p} f\left(x^{\prime} p^{\prime}\right)\right|+ \\
& +\left|e^{\beta(x) p} \int_{\left(x^{\prime} p^{\prime}\right)}^{(x p)} d t I[F \mid F]\left(x_{t} p_{t}\right)\right| \stackrel{(\alpha)}{\leqq} \mid e^{\beta\left(x^{\prime}\right) p^{\prime} f\left(x^{\prime} p^{\prime}\right) \mid} \\
& +\left|\int_{\left(x^{\prime} p^{\prime}\right)}^{(x p)} d t e^{\beta\left(x_{t}\right) p_{t}} I[F \mid F]\left(x_{t} p_{t}\right)\right| \leqq A+ \\
& +\int_{\left(x^{\prime} p^{\prime}\right)}^{(x p)} d t \iiint\left\{\left|F^{\prime}(x 3) e^{+\beta 3} F(x 4) e^{+\beta 4}\right|+\left|F\left(x p_{t}\right) e^{+\beta p} t F(x 2) e^{\beta 2}\right|\right\} e^{-\beta 2} \times \\
& \times \delta(p+2-3-4) \times p_{a} 2^{a} \times \sigma(p 2 \rightarrow 34) d 234 \leqq \\
& \stackrel{(\gamma)}{\leqq} A+8 A^{2} \int_{\left(\mathrm{x}^{\prime} p^{\prime}\right)}^{(\mathrm{xp})} d t \int_{2} \sigma p_{a} 2^{a} \times e^{-\beta 2} d 2 \stackrel{(\delta)}{\leqq} A+8 A^{2} \sigma B . \\
& \|\tilde{F}\| \leqq A+8 A^{2} \sigma B .
\end{aligned}
$$

So,

The steps in this over-estimation are justified as follows: $(\alpha)$ by the fact that $e^{\beta p}$ does not increase with $t$. $(I[F \mid F]$ stands for the 3-fold integral in (6)). ( $\beta$ ) by (9), (7) and the fact that $e^{\beta 1}=e^{\beta 3} e^{\beta 4} e^{-\beta 2}$ on the collision manifold; $(\gamma)$ by $(10)$ and the definition (14) of $\Phi$; finally $(\delta)$ by the definition (13) of $W$.

d) An estimation of $\|\tilde{F}-\tilde{G}\|$ for two functions $F, G \in \Phi$ proceeds exactly in the same way and need not be reproduced; its result is

$$
\|\tilde{F}-\tilde{G}\| \leqq 4 A B \sigma\|F-G\| \text {. }
$$

(17) and (18) show that if we choose for $B$ the number $\frac{1}{8 A \sigma}$, then maps $\Phi$ into itself and is contractive: $\|\tilde{F}-\tilde{G}\| \leqq 1 / 2\|F-G\|$, provided, at least, the map is well defined and yields measurable functions $\tilde{F}$. To show this, will be the content of the next section.

e) ${ }^{12}$ We observe first that the function $(x 12) \rightarrow F(x 1) F(x 2)$ is measurable with respect to $\sigma k d X d 1 d 2$ as a product of functions $(x 12) \rightarrow F(x 1)$. Because of (14), the function $(x 12) \rightarrow F(x 1) F(x 2)$ is even integrable.

Let $S$ be the set of points $(x 1234)$ with $1+2-3-4=0$ and let $d s=\sigma(12) k(12) \delta(1+2-3-4) d X d 1 d 2 d 3 d 4$. The measure $d s$ on $S$ is an integral

$$
d s=\int d X d 1 d 2\left\{(x 12) \rightarrow \delta_{x} \sigma k \delta(1+2-3-4) d 3 d 4\right\}
$$

12 For this section ef. Bourbakr, integration ch. V, and Gelfand-SHILov, 1960. 
of measures $\delta_{x} k \sigma \delta d 3 d 4$ and this representation of $d s$ is vaguely continuous. Next we observe that the function $(x 1234) \rightarrow F(x 1) F(x 2)$ on $S$ is integrable with respect to $d s$. For there exist continuous $d X d 1 d 2$ integrable functions $\psi_{1}(x 12), \psi_{2}(x 12)$ with $\psi_{1}(x 12) \leqq F(x 1) F(x 2) \leqq$ $\leqq \psi_{2}(x 12)$ and $\int\left|\psi_{1}-\psi_{2}\right| \sigma k d X d 1 d 2$ arbitrarily small, say smaller than $\varepsilon$. Then the functions $\tilde{\psi}_{1}:(x 1234) \rightarrow \psi_{1}(x 12)$ on $S$ are continuous bounds for $(x 1234) \rightarrow F(x 1) F(x 2)$, integrable with respect to $d s$ and satisfy $\int\left|\tilde{\psi}_{1}-\tilde{\psi}_{2}\right| d s<\varepsilon$. One sees in the same way that the function $(x 1234) \rightarrow F(x 3) F(x 4)$ is $d s$-integrable.

Now, because of the vague continuity of the representation (19),

$$
\iint F(x 3) F^{\prime}(x 4) \sigma(12 \rightarrow 34) k(12) \delta(1+2-3-4) d 3 d 4
$$

exists for $d X d 1 d 2$-almost-all $(x 12)$ and is $x 12$-integrable. By Fubini's theorem, $\iiint F(x 3) F(x 4) \sigma k \delta(1+2-3-4) d 2 d 3 d 4$ exists $d X d 1$ almost-everywhere and is integrable, hence measurable. An even simpler argument holds for the second term of the collision integral in (6) and yields the existence and integrability of $I[F \mid F]$. Now, according to $(5), \int I[F \mid F] d \tau=\int I[F \mid F] d t \wedge \omega$, so $\int I[F \mid F] d t$ exists almost everywhere and is measurable ${ }^{13}$.

f) The existence of a solution is now established. Its uniqueness in the class $\Phi$ follows immediately from the uniqueness statement in Banach's fixed point theorem. There could, however, still exist a solution which is exponentially bounded by an exponential $e^{-\beta^{\prime} p}$ other than the one we chose. But then, according to the lemma, there exists a bound $e^{-\beta p}$ which is greater than $e^{-\beta^{\prime} p}$, hence both of our assumed solutions lie in the space $\Phi$ defined with $e^{-\beta p}$ and thus are equal. Note that uniqueness means always uniqueness up to a set of measure zero.

g) In order to prove positivity of the solution for positive $f$, consider the same time layer $W$ as in a) and the set $\Phi+$ of positive (a.e.) functions of $\Phi$. Define a mapping ^ of $\Phi+$ into $\Phi+$ by

$$
\hat{F}=\max (0, \tilde{F}), \quad F \in \Phi+.
$$

The same arguments as for $\sim$ show that $\_$is indeed a mapping of $\Phi+$ into $\Phi+$, again contractive. So , has a fixed point. It has, by modification on a set of measure zero, a fixed point $F$ which is continuous along geodesics. We show that $F=\tilde{F}$ a.e., such that $F$ is also a fixed point of $\sim$ and hence a solution of the Boltzmann equation. For if $\hat{F} \neq F=\hat{F}$, then $\tilde{F}<0$ in a point $(x p)$. Then there is on the geodesic through $(x p)$ an earliest point $\left(x^{\prime} p^{\prime}\right)$ such that $F\left(x^{\prime} p^{\prime}\right)=\tilde{F}\left(x^{\prime} p^{\prime}\right)=\hat{F}\left(x^{\prime} p^{\prime}\right)=0$ and such that $\tilde{F}<0$, and hence $F=0$, for all later points which are sufficiently near to $\left(x^{\prime} p^{\prime}\right)$. But then an inspection of (12) shows that $\tilde{F}$ does

${ }_{13} \int I d t$ is, in fact, an indeterminate integral. But an obvious "triangle argument" makes Fubini's theorem applicable. 
not decrease from $\left(x^{\prime} p^{\prime}\right)$ on, the negative term in the collision integral being zero, which is a contradiction. Hence, $F$ coincides (a.e.) with the unique fixed point of $\sim$.

\section{Indication of Further Results}

1) If the initial distribution $f$ is positive and bounded also from below by a local distribution function $\bar{A} e^{-\beta^{\prime} p}$ on $H$, then it will remain so on $W$. To show this, one constructs a continuous vector field $\bar{\beta}$ on $D$ which continues $\bar{\beta}^{\prime}$ and is such that the function $\bar{\beta}_{a} p^{a}$ does not decrease along phase paths. The proof of the existence of $\bar{\beta}$ is a straightforward analog of the proof of the lemma. Now, the subset of $\Phi$ consisting of all functions greater than $1 / 2 \bar{A} e^{-\beta p}$ is shown to be closed and invariant under $\sim$, so the fixed point of $\sim$ lies in it. This persistence property is another hint that a distribution stays close to local equilibrium, a condition necessary for the Chapman-Enskog method to make sense (cf. remark 2 , Sec. II). By a similar argument on closed invariant subsets it can be shown that if $f$ happens to be continuous, so is $F$.

2) To prove local existence and uniqueness we simply carried over the well-known Picard-Lindelof procedure in the theory of ordinary differential equations. As is done there, we can refine the method to study also the dependence of the solution on the initial distribution and on the ingredients of the equation, viz. $F_{b}^{a}, \Gamma_{b c}^{a}, \sigma(12 \rightarrow 34)$. The way to do it is obvious, though lengthy, and yields the following:

If the initial distributions $f_{n}$ converge to $f$ in the norm

$$
\|f\|=\text { (ess. } \max .\left|e^{\beta^{\prime} p^{\prime}} f\left(x^{\prime} p^{\prime}\right)\right|, x^{\prime} \in H, \omega \text { the measure), }
$$

then the corresponding solutions $F_{n}$ and $F$ exist on a common time layer and $F_{n} \rightarrow F$ in the (pseudo-)metric (15). If $f$ is continuous, and $F_{n b}{ }^{a}{ }_{b} F^{a}{ }_{b}$ and $\Gamma_{n}{ }_{b c} \rightarrow \Gamma_{b c}^{a}$ uniformly on a neighborhood of $H$, then there exists a common time layer for the corresponding solutions $F_{n}, F$ and $F_{n} \rightarrow F$ in the metric (15). Finally, the same behavior of the respective solutions holds if $\max _{1,2} \int\left|\sigma_{n}(12 \rightarrow 34)-\sigma(12 \rightarrow 34)\right| d 3 d 4 \rightarrow 0$.

I would like to express my thanks to Professor Jürgen EHLERs for many stimulating discussions in connection with this paper.

\section{References}

Bichteler, K.: Bemerkungen über relativistische Stoßinvarianten. Z. Physik 182, $521-523$ (1964).

- Beiträge zur relativistischen kinetischen Gastheorie. Dissertation Hamburg 1965.

Bishop, R. L., and R. J. Crittenden: Geometry of manifolds. New York: Academic Press 1964.

Bourbaki, N.: Integration. Ch. V. Paris: Herrmann 1960. 
Chernikov. N. A.: Acta Phys. Polon. 23, 629 (1963) (and earlier papers cited therein).

EHLERs, J.: Beiträge zur relativistischen Mechanik relativistischer Medien. Abhandl. Akad. Wiss. Mainz, Math.-Naturw. Klasse Nr. 11, 1961.

Gelfand, I. M., u. G. E. ShILov: Verallgemeinerte Funktionen. Berlin: VEB Verlag der Wissenschaften 1960.

KaMkE, E.: Differentialgleichungen reeller Funktionen. Leipzig: Geest \& Portig KG 1956.

Lichnerowicz, A.: Theorie globale des connexions et des groupes d'holonomie. Roma: Edizione Cremonese 1955.

LINDQUist, R. W.: Relativistic transport theory. Ann. Phys. 37, 487-518 (1966).

TAUber, E. S., and J. W. WeInBerg: Phys. Rev. 122, 4 (1961). 